\title{
Cooperative Wireless-Based Obstacle/Object Mapping and See-Through Capabilities in Robotic Networks
}

\author{
Yasamin Mostofi, Member, IEEE
}

\begin{abstract}
In this paper, we develop a theoretical and experimental framework for the mapping of obstacles (including occluded ones), in a robotic cooperative network, based on a small number of wireless channel measurements. This would allow the robots to map an area before entering it. We consider three approaches based on coordinated space, random space, and frequency sampling, and show how the robots can exploit the sparse representation of the map in space, wavelet or spatial variations, in order to build it with minimal sensing. We then show the underlying tradeoffs of all the possible sampling, sparsity and reconstruction techniques. Our simulation and experimental results show the feasibility and performance of the proposed framework. More specifically, using our experimental robotic platform, we show preliminary results in successfully mapping a number of real obstacles and having see-through capabilities with real structures, despite the practical challenges presented by multipath fading.
\end{abstract}

Index Terms-Autonomous robots, obstacle mapping, wireless communications, compressive sensing

\section{INTRODUCTION}

$\mathrm{M}$ OBILE intelligent networks can play a key role in many different areas from emergency response, surveillance and security, and battlefield operations to smart homes and factories and environmental monitoring [2], [3], [4]. The vision of a multiagent robotic network cooperatively learning and adapting in harsh unknown environments to achieve a common goal is closer than ever.

An important issue key to the robust operation of a mobile robotic network is the accurate mapping of the obstacles. Yet the high volume of the information presented by the environment makes it prohibitive to sense all the areas, making accurate mapping challenging. In most related work, only areas that are directly sensed are mapped. The rich literature on Simultaneous Localization and Mapping (SLAM) and its several variations fall into this category [5], [6], [7], [8]. SLAM approaches mainly focus on reducing the uncertainty in the sensed landmarks, by using a Kalman filter. Similarly, approaches based on generating an occupancy map also address sensing uncertainty [9]. Another set of approaches are based on the Next Best View (NBV) problem [10], [11], [12], [13], [14]. In NBV approaches, the aim is to move to the positions that are "good" for sensing, by guiding the vehicles to the perceived next safest area (area with the most visibility) based on the current map [10]. However, areas that are not sensed directly are not mapped in NBV.

In this paper, we are interested in cooperative obstacle/ object mapping and see-through capabilities, i.e., mapping

- The author is with the Department of Electrical and Computer Engineering, University of California Santa Barbara, Santa Barbara, CA 93106. E-mail: ymostofi@ece.ucsb.edu.

Manuscript received 18 June 2011; revised 24 Dec. 2011; accepted 30 Dec. 2011; published online 23 Jan. 2012.

For information on obtaining reprints of this article, please send e-mail to: tmc@computer.org, and reference IEEECS Log Number TMC-2011-06-0325. Digital Object Identifier no. 10.1109/TMC.2012.32. of occluded obstacles. By obstacle/object mapping, we refer to building a spatial map of the obstacles or objects in the environment, where a nonzero value, at any position, indicates that there is an obstacle there, while a zero indicates otherwise. By cooperative obstacle/object mapping, we refer to a number of robots cooperating to generate a spatial map of the obstacles. Finally, by having seethrough capabilities, we refer to an approach that can map occluded obstacles. Consider the case where a number of robots want to build a map of the obstacles inside a building before entering it. Having see-through capabilities allows the nodes to assess the situation before entering the building and can be of particular interest in several applications such as emergency response, surveillance, security or battlefield operation [15]. It furthermore allows the robots to map the obstacles for navigation purposes, without having to sense them directly. In general, devising see-through mapping strategies can be considerably challenging and cannot be achieved with the traditional sensing and mapping techniques.

In this paper, we develop a framework for the cooperative mapping of obstacles (including occluded ones) in robotic networks, by only using a small number of wireless transmissions and utilizing the new theories of compressive sensing. Using wireless transmissions allows us to map the areas that are not directly sensed. However, it is very challenging to extract obstacle information from a wireless reception, without making a prohibitive number of measurements, due to several propagation phenomena. We then show how to properly develop the necessary theoretical and experimental framework to extract this information, based on a small number of wireless measurements, by 1) using the recent breakthroughs in nonuniform sampling theory [16], [17] and 2) properly designing a robotic platform to limit multipath fading in practice.

A survey of the existing work in the literature shows few papers that use wireless measurements for the cooperative 
mapping of occluded obstacles in robotic networks. There are, however, a number of papers on detecting an object, using fixed sensors. For instance, in [15], authors use a transmitter that is buried underground and several fixed receivers on the surface to detect underground tunnels/ facilities using radar. In [18], [19], the authors build a network of 28 fixed sensors in order to track a person, based on making several measurements between pairs of sensors. In [20], [21], we discussed the original idea that compressive sensing can be used for estimating a spatial field in three different applications: aerial mapping, mapping of a communication field to a fixed transmitter, and mapping of obstacles. In the area of obstacle mapping, the part relevant to this paper, that paper introduced the initial idea that wireless transmissions can be used for cooperative obstacle mapping in robotic networks, where we proposed mapping based on frequency sampling. In this paper, we extend our previous work to develop a comprehensive theoretical and experimental framework for wireless-based obstacle mapping based on different sampling, sparsity and reconstruction approaches.

Let an obstacle map refer to a 2D (or 3D) map of the environment, where we have zeros at locations where there are no obstacles and nonzero values at obstacle locations. Each nonzero value, for instance, could be the decay rate of the wireless signal within the object at that location, as we shall see in Section 3. In this work, we present a comprehensive foundation for obstacle mapping, based on a small number of wireless measurements and develop the necessary robotic experimental setup so that the map can be extracted, with minimal sensing, despite the practical challenges presented by multipath fading. More specifically, we propose three approaches based on coordinated space, random space, and frequency sampling. We show how the vehicles can utilize the sparse representation of the map in space, wavelet, or spatial variations, in order to reconstruct the map cooperatively, based on minimal measurements, and more importantly with a seethrough capability. We develop a comprehensive framework to shed light on the underlying tradeoffs of different sampling, sparsity, and reconstruction techniques. We furthermore show how to properly develop an experimental robotic setup, including antenna control, to implement the proposed approach. We then use our experimental setup and show the performance of our framework in efficiently mapping a number of real obstacles (including completely blocked ones), with their details, based on only very few wireless channel measurements. We emphasize that we do not view this framework as a replacement for the current obstacle sensing and mapping techniques. We rather view it as a complement to the existing approaches, by providing a see-through capability.

The paper is organized as follows: In Section 2, we provide an overview of compressive sampling theory and different relevant reconstruction techniques. In Section 3, we show how wireless measurements can be used for obstacle mapping. In Section 4, we propose different approaches for sampling, sparsity, and reconstruction of an obstacle map and show the underlying tradeoffs. Section 5 then discusses our experimental platform and shows the performance of our framework in mapping a number of real structures. We conclude in Section 6.

\section{An Overview of Compressive Sampling THEORY [16], [17], [22]}

Consider the case where we are interested in recovering a vector $x \in \mathbb{R}^{N}$. For $2 \mathrm{D}$ signals, vector $x$ can represent the columns of the matrix of interest stacked up to form a vector. Let $y \in \mathbb{R}^{K}$ represent the incomplete linear measurement of vector $x$, obtained by the robots, where $K \ll N$. We have $y=\Phi x$, where we refer to $\Phi$ as the observation matrix. Solving for $x$ based on the observation set $y$ is an ill-posed problem as the system is severely underdetermined $(K \ll N)$. However, consider the case where $x$ has a sparse representation in another domain, i.e., it can be represented as a linear combination of a small set of vectors: $x=\Gamma X$, where $\Gamma$ is an invertible matrix and $X$ is S-sparse: $|\operatorname{supp}(X)|=S \ll N$, with $\operatorname{supp}(X)$ referring to the set of indices of the nonzero elements of $X$ and $|\cdot|$ denoting its cardinality. This means that the number of nonzero elements in $X$ is considerably smaller than $N$. Then, we have $y=\Psi X$, where $\Psi=\Phi \times \Gamma$. In general, we do not know anything about the structure of $X$ except for the fact that it is sparse (which we can validate by analyzing similar data). The new theory of compressive sensing allows us to solve this problem.

Theorem 1 (see [16]). If $K \geq 2 S$ and under specific conditions, the desired $X$ is the solution to the following optimization problem: $\min \|X\|_{0}$, such that $y=\Psi X$, where $\|X\|_{0}=|\operatorname{supp}(X)|$ represents the zero norm of vector $X$. Thus, we only need $2 \times S$ measurements to recover $X$ and therefore $x$ fully. However, we need to solve a nonconvex combinatorial problem, which is not practical.

Theorem 2 (see [16], [17]). Assume that $X$ is S-sparse. The following $\ell_{1}$ relaxation: $\min \|X\|_{1}$, subject to $y=\Psi X$, can exactly recover $X$ from measurement $y$ if matrix $\Psi$ satisfies the Restricted Isometry Condition (RIC) for $(2 S, \sqrt{2}-1)$.

The RIC condition is mathematically related to the uncertainty principle of harmonic analysis [22].

It has furthermore been shown that random partial Fourier matrices as well as random Gaussian or Bernoulli matrices satisfy RIC (a stronger version) with the probability $1-O\left(N^{-M}\right)$ if $K \geq B_{M} S \times \log ^{O(1)} N$, where $B_{M}$ is a constant, $M$ is an accuracy parameter and $\mathrm{O}(\cdot)$ is Big-O notation [16]. This shows that the number of required measurements could be considerably less than $N$.

\subsection{Basis Pursuit (BP): Reconstruction Using $\ell_{1}$ Relaxation}

The compressive sensing algorithms that reconstruct the signal based on the $\ell_{1}$ relaxation of Theorem 2 are referred to as "Basis Pursuit" [17]. Reconstruction through $\ell_{1}$ optimization has the strongest known recovery guarantees [22]. Sparse Reconstruction by Separable Approximation (SpaRSA) is an example of a relatively new solver, based on $\ell_{1}$ relaxation, which we find to be computationally efficient, yet effective, and will use in the subsequent sections [23].

\subsection{Matching Pursuit (MP): Reconstruction Using Successive Interference Cancellation [22], [24], [25]}

While the $\ell_{1}$ relaxation of the previous part can solve the compressive sampling problem with performance 
guarantees, its computational complexity can be high. Alternatively, there are greedy approaches that can solve the compressive sampling problem more efficiently, at the cost of a possible loss of performance. Next, we summarize such approaches. The Restricted Isometry Condition implies that the columns of matrix $\Psi$ should have a certain near-orthogonality property. Let $\Psi=\left[\Psi_{1} \Psi_{2} \cdots \Psi_{N}\right]$, where $\Psi_{i}$ represents the $i$ th column of matrix $\Psi$. We will have $y=\sum_{j=1}^{N} \Psi_{j} X_{j}$, where $X_{j}$ is the $j$ th component of vector $X$. Consider recovering $X_{i}$ :

$$
\frac{\Psi_{i}^{H} y}{\Psi_{i}^{H} \Psi_{i}}=\underbrace{X_{i}}_{\text {desired term }}+\underbrace{\sum_{j=1, j \neq i}^{N} \frac{\Psi_{i}^{H} \Psi_{j}}{\Psi_{i}^{H} \Psi_{i}} X_{j}}_{\text {interference }} .
$$

Orthogonal Matching Pursuit (OMP) iteratively multiplies the measurement vector, $y$, by $\Psi^{H}$, recovers the strongest component, subtracts its effect and continues again [24]. Let $I_{\text {set }}$ denote the set of indices of the nonzero coefficients of $X$ that is estimated and updated in every iteration. Once the locations of the $S$ nonzero components of $X$ are found, we can solve directly for $X$ by using a least squares solver: $\hat{X}=\operatorname{argmin}_{X: \operatorname{supp}(X)=I_{\text {set }}}\|y-\Psi X\|_{2}$. A variation of OMP, Regularized Orthogonal Matching Pursuit (ROMP), was later introduced [22]. In ROMP, a set of indices (locations of vector $X$ with nonnegligible components) are recovered at the same time at every step, instead of only one at a time, resulting in a faster recovery. Readers are referred to [22], [24], [25] for more details. In this paper, we also use MP approaches for reconstruction in our mapping framework and show the underlying tradeoffs.

\subsection{Reconstruction Using Total Variation (TV) Minimization}

Consider an obstacle map. The spatial variations of the map (gradient) are also considerably sparse. Thus, another related sparsity-based reconstruction approach is to use the sparsity in the gradient. In this part, we briefly discuss reconstruction based on gradient sparsity [16], [26], [27]. Let $\mathcal{F}=\left[\mathcal{F}_{i, j}\right]$ denote an $m \times m$ matrix that represents the spatial function of interest. Define the following operators:

$$
\begin{aligned}
& D_{h, i, j}(\mathcal{F})= \begin{cases}\mathcal{F}_{i+1, j}-\mathcal{F}_{i, j} & i<m \\
0 & i=0,\end{cases} \\
& D_{v, i, j}(\mathcal{F})= \begin{cases}\mathcal{F}_{i, j+1}-\mathcal{F}_{i, j} & j<m \\
0 & j=0,\end{cases}
\end{aligned}
$$

and

$$
D_{i, j}(\mathcal{F})=\left(\begin{array}{c}
D_{h, i, j}(\mathcal{F}) \\
D_{v, i, j}(\mathcal{F})
\end{array}\right)
$$

Then, the Total Variation function is defined as follows:

$$
\begin{aligned}
\operatorname{TV}(\mathcal{F}) & \triangleq \sum_{i} \sum_{j} \sqrt{\left(D_{h, i, j}(\mathcal{F})\right)^{2}+\left(D_{v, i, j}(\mathcal{F})\right)^{2}} \\
& =\sum_{i j}\left\|D_{i, j}(\mathcal{F})\right\|_{2} .
\end{aligned}
$$

TV minimization approaches solve the following problem or a variation of it: $\min \operatorname{TV}(\mathcal{F})$, subject to $y=\Psi_{\mathcal{F}} \times V_{\mathcal{F}}$, where $V_{\mathcal{F}}$ is a column vector that results from stacking up the columns of matrix $\mathcal{F}$, and $y$ is the observation vector, which is linearly related to $V_{\mathcal{F}}$ through matrix $\Psi_{\mathcal{F}}$. Recently, TVAL [27] is proposed for solving this problem more efficiently and robustly. In the next section, we also use TVbased approaches for reconstructing an obstacle map. We use the terms "space TV" or "TV" to refer to the spatial variations of the map throughout the paper. Consequently, "TV sparsity" refers to utilizing the sparsity of the spatial variations for reconstruction. We emphasize that sparsity in TV is different from sparsity in space as the former is the sparsity of variations while the latter is the sparsity of the map directly.

\section{Problem Setup: Cooperative Mapping of Obstacles Using Wireless Measurements}

In this section, we show how a group of mobile nodes can build a map of obstacles and have see-through capabilities [21]. More specifically, we formulate the problem setup to express a wireless transmission as a function of obstacle information. We consider building a $2 \mathrm{D}$ map of the obstacles in this paper. For instance, for real 3D structures, we reconstruct a horizontal cut of them, as shown in Section 5. It should be noted that our approach can also be extended to 3D maps, as we shall discuss in Section 6. Fig. 1 (both left and right) shows a sample 2D obstacle map and illustrates the considered problem, i.e., a number of vehicles want to map the space before entering it, using wireless measurements. As mentioned in Section 1, let an obstacle map refer to a 2D (or 3D) map of the environment, where we have zeros at locations where there are no obstacles and nonzero values at obstacle locations. Each nonzero value could be the decay rate of the wireless signal within the object at that location. Let $g(u, v)$ represent the obstacle map at position $(u, v) \in \mathbb{R}^{2}$. We have

$$
g(u, v)= \begin{cases}\eta(u, v) & \text { if }(u, v) \text { is an obstacle } \\ 0 & \text { else }\end{cases}
$$

where $\eta(u, v)<0$ denotes the decay rate of the wireless signal inside the object at position $(u, v) \in \mathbb{R}^{2}$. Note that, in general, $\eta(u, v)$ is space varying since the structure may not be homogeneous. Similarly, let $g_{\mathrm{bin}}(u, v)$ represent the binary version of the obstacle map at position $(u, v) \in \mathbb{R}^{2}$. We have

$$
g_{\text {bin }}(u, v)= \begin{cases}1 & \text { if }(u, v) \text { is an obstacle } \\ 0 & \text { else. }\end{cases}
$$

Consider the communication from Transmitter 1 to Receiver 1, as marked in Fig. 1 (left). This communication contains implicit information of the obstacles along the communication path. Consider the dashed ray (line) that is at distance $t$ from the origin, and perpendicular to the line that is at angle $\theta$ with the $x$-axis in Fig. 1 (left). Let $P(\theta, t)$ represent the received signal power in the transmission along this line. We have the following [21], [28], [29],

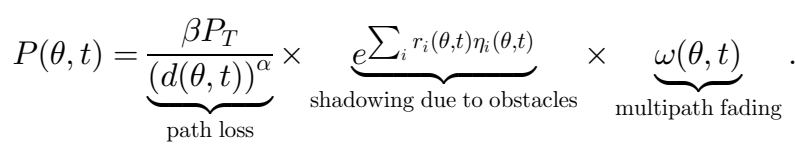

For the path loss term, $P_{T}$ represents the transmitted power, $d(\theta, t)$ is the distance between the transmitter and receiver across that ray, $\alpha$ is the degradation exponent and 

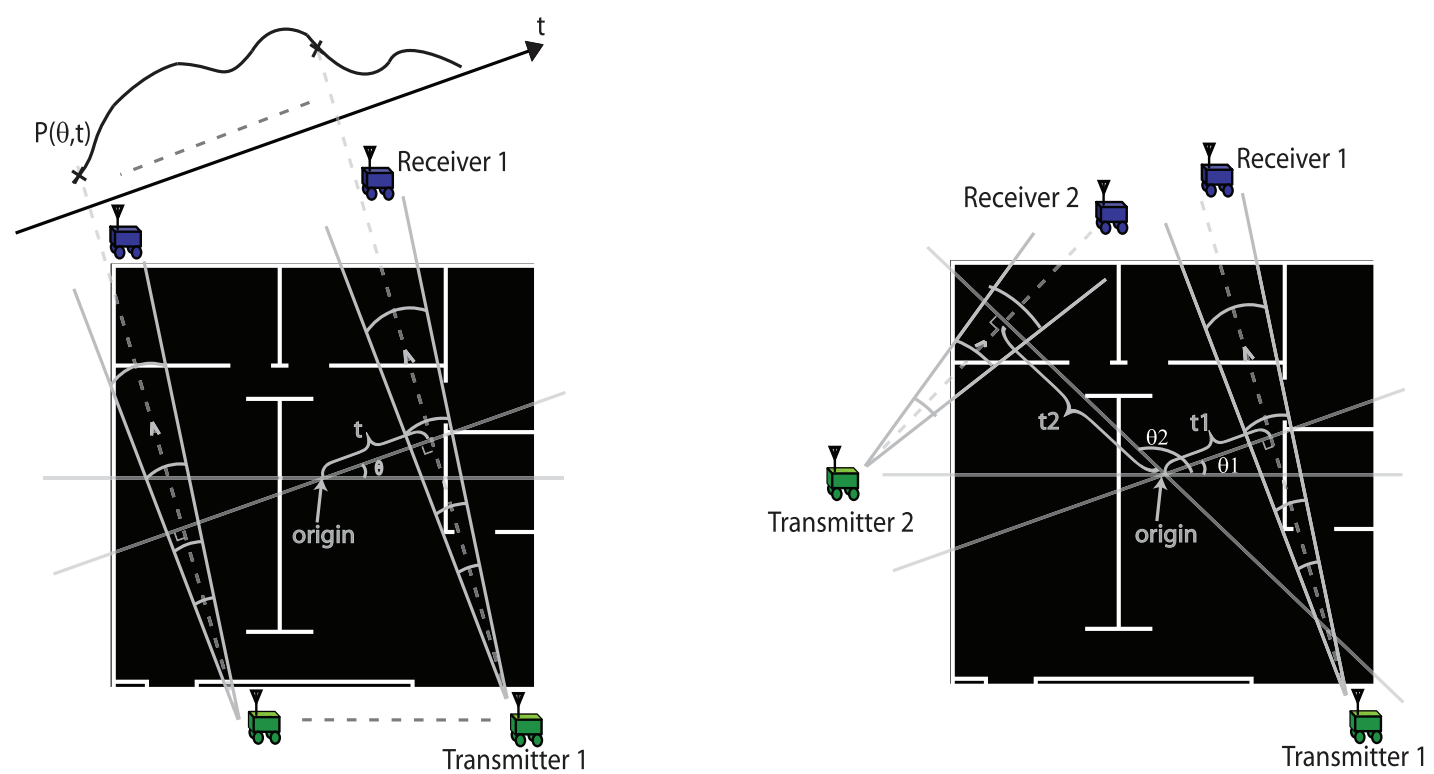

Fig. 1. An indoor obstacle map with the obstacles marked in white and the illustration of the considered mapping problem, using coordinated (left) and random (right) wireless measurements.

$\beta$ is a constant that is a function of system parameters. For the shadowing (or shadow fading) term, $r_{i}$ is the distance traveled across the $i$ th object along the $(\theta, t)$ ray and $\eta_{i}<0$ is the decay rate of the wireless signal within the $i$ th object. Furthermore, the summation is over the objects across that line. As can be seen, shadowing characterizes wireless signal attenuation as it goes through the obstacles along the transmission path and therefore contains information about the objects along that line. $\omega(\theta, t)$, on the other hand, is a positive random variable with unit average, which models the impact of multipath fading. For more mathematical details on wireless channel modeling, readers are referred to [28], [29], [30]. We then have

$$
\begin{aligned}
h(\theta, t) & \triangleq \ln P(\theta, t)-\ln P_{T}-\left(\beta_{\mathrm{dB}}-\alpha \ln d(\theta, t)\right) \\
& =\underbrace{\sum_{i} r_{i}(\theta, t) \eta_{i}(\theta, t)}_{\text {shadowing due to blocking objects in dB }}+\underbrace{\omega_{\mathrm{dB}}(\theta, t),}_{\text {multipath fading in } \mathrm{dB}}
\end{aligned}
$$

where $\beta_{\mathrm{dB}}=\ln \beta$ and $\omega_{\mathrm{dB}}=\ln \omega(\theta, t)$. Path loss and shadowing represent the signal degradation due to the distance traveled and obstacles, respectively, and $\omega_{\mathrm{dB}}(\theta, t)$ represents the impact of multipath fading. By using an integration over the line that corresponds to $\theta$ and $t$, we can express (3) as follows:

$$
h(\theta, t)=\iint_{\operatorname{line}(\theta, t)} g(u, v) d u d v+\omega_{\mathrm{dB}}(\theta, t),
$$

where $g(u, v)$ is the obstacle map of (1). As can be seen, $h(\theta, t)$, obtained through wireless measurements, contains implicit information on the obstacle map. ${ }^{1}$ This is the foundation of our framework. More specifically, we show different ways of extracting the obstacle map from a small number of wireless measurements, as we shall see in the next section. The multipath fading term presents a

1. In practice, the path loss component can be estimated by using a few Line Of Sight (LOS) transmissions in the same environment. Therefore, its impact can be removed and the receiving robot can calculate $h(\theta, t)$. considerable challenge in extracting the map. We also show how a proper design and control of TX/RX antennas can help reduce the impact of multipath fading, making mapping based on wireless transmissions feasible.

\section{Different Possibilities for Compressive SAMPLING AND RECONSTRUCTION}

In this section, we discuss the details of our framework for compressive obstacle mapping, using wireless measurements. More specifically, we propose different possibilities in terms of 1) sampling, 2) sparsity domain, and 3) reconstruction technique. A summary of our framework is shown in Fig. 2. Note that not all the combinations of the figure result in a proper problem formulation, as we shall discuss in the paper.

\subsection{Frequency Sampling Using Coordinated Wireless Measurements}

In this part, we discuss an obstacle mapping approach that is motivated by computed tomography approaches in medical imaging, geology, and computer graphics [31], [32], [33]. In [21], we introduced the original idea. In this section, we expand it by utilizing the sparsity in wavelet and total variations, and showing the impact of nonideal frequency sampling. In the subsequent sections, we introduce our other proposed sampling/sparsity strategies. Then, we develop a comprehensive framework to compare the performance of

Elements of Compressive Cooperative Obstacle Mapping

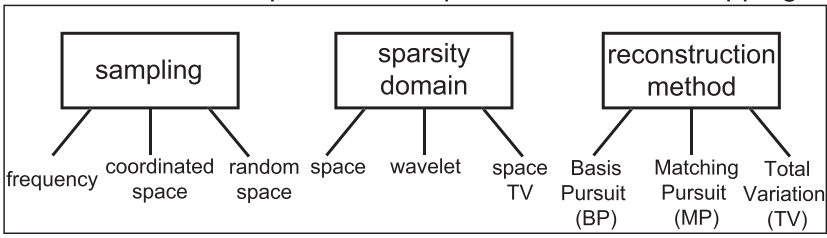

Fig. 2. Elements of our proposed framework for compressive cooperative obstacle mapping. 
all these approaches and show the underlying tradeoffs, using both simulation and experiment.

Consider Fig. 1 (left), where pairs of robots are making coordinated wireless measurements. Consider the illustrated line at angle $\theta$ that passes through the origin. Outside the structure, two robots can move parallel to this line, in a coordinated fashion, such that a number of wireless channel measurements are formed at different $t \mathrm{~s}$. By changing $t$ at a specific $\theta$, a projection is formed $(P(\theta, t)$ for a set of $t$ s), i.e., a set of ray integrals, as is shown in Fig. 1 (left).

Let $G_{f}\left(\theta_{f}, f\right)$ represent the 2D Fourier transform of $g$, expressed in the polar coordinates, where $\theta_{f}$ is the angle from the $x$-axis and $f$ is the distance from the origin. Let $H_{t}(\theta, f)$ denote the 1D Fourier transform of $h(\theta, t)$ with respect to $t: H_{t}(\theta, f)=\int h(\theta, t) e^{-j 2 \pi f t} d t$. The following theorem allows us to sample the frequency response of the $2 \mathrm{D}$ obstacle map, using a projection, i.e., based on gathering coordinated wireless measurements.

Fourier slice theorem: Consider the case where there is no multipath fading in (4), i.e., $\omega_{\mathrm{dB}}=0$. Then, $H_{t}(\theta, f)$, the Fourier transformation of $h(\theta, t)$ with respect to $t$, is equal to the samples of $G_{f}\left(\theta_{f}, f\right)$ across angle $\theta_{f}=\theta$.

Proof. See [31].

By making a number of measurements at different $t$ s for a given $\theta$, the Fourier Slice Theorem allows us to measure the samples of the Fourier transform of the map at angle $\theta$. By changing $\theta$, we can sample the Fourier transform of the obstacle map at different angles. We can then pose the problem in a compressive sampling framework. By measuring the received signal power across a number of rays, the vehicles can indirectly sample the Fourier transformation of the obstacle map. Then, the sparsity in the space, space TV or wavelet domain could be used for reconstruction, as explained next.

\subsubsection{Reconstruction Using the Sparsity in Space or TV}

Let $V_{G_{f}}$ denote the vector representation of the discrete version of $G_{f}$ (2D Fourier transform of the obstacle map), where the columns are stacked up to form a vector. Let $y_{f}$ represent the very few samples of $G_{f}$ acquired through wireless channel measurements, across a number of coordinated rays, and applying the Fourier Slice Theorem. We have

$$
\begin{aligned}
y_{f}= & \Phi_{\mathrm{pt}} V_{G_{f}} \text { and } V_{G_{f}}=\Gamma_{f} V_{g_{s}} \Rightarrow y_{f}=\Psi_{f, s} V_{g_{s}} \\
& \text { Fourier Sampling and space or TV sparsity, }
\end{aligned}
$$

where $\Phi_{\mathrm{pt}}$ is a point sampling matrix:

$$
\begin{aligned}
& \forall i 1 \leq i \leq K, \exists j 1 \leq j \leq N \text { such that } \Phi_{\mathrm{pt}}(i, j)=1 \text { and } \\
& \forall i 1 \leq i \leq K, \forall j \neq j^{\prime}, 1 \leq j, j^{\prime} \leq N, \\
& \quad \text { if } \Phi_{\mathrm{pt}}(i, j)=1 \rightarrow \Phi_{\mathrm{pt}}\left(i, j^{\prime}\right)=0,
\end{aligned}
$$

with $K$ and $N$ denoting the sizes of $y_{f}$ and $V_{G_{f}}$, respectively. Matrix $\Phi_{\text {pt }}$ represents a matrix with only one 1 in every row. If there are redundant measurements, there may be more than one 1 in every column. Otherwise, there will be at most one 1 in every column. Let $g_{s}$ represent the discrete obstacle map (the discrete version of $g$ of (1)). Then, $V_{g_{s}}$ denotes the vector representation of $g_{s}$ and $\Gamma_{f}$ is the Fourier transform matrix, such that when applied to a vector that is formed by stacking the columns of a $2 \mathrm{D}$ map, it results in the vector representation of the 2D Fourier transform of the map. We have $\Psi_{f, s} \triangleq \Phi_{\mathrm{pt}} \times \Gamma_{f}$. Such matrices meet the RIC condition, as shown in [34]. Thus, we expect a good reconstruction quality with this approach. Equation (5) can then be solved by any of the compressive sensing approaches of the previous section (BP or MP), by using the sparsity in the space domain. Alternatively, we can consider the sparsity in the Total Variation of $V_{g_{s}}$. In the next section, we show the underlying tradeoffs between these approaches.

\subsubsection{Reconstruction Using the Sparsity in the Wavelet Domain}

Typically, an obstacle map is also considerably sparse in the wavelet domain, as we shall see later in this section. Let $G_{w}$ represent the 2D wavelet transform matrix of the discrete obstacle map $g_{s}$. We have

$$
\begin{aligned}
y_{f}= & \Phi_{\mathrm{pt}} V_{G_{f}} \text { and } V_{G_{f}}=\Gamma_{w} V_{G_{w}} \Rightarrow y_{f}=\Psi_{f, w} V_{G_{w}} \\
& \text { Fourier sampling and wavelet sparsity, }
\end{aligned}
$$

where $\Gamma_{w}=\Gamma_{f} \times W^{-1}$ with $W$ representing a $2 \mathrm{D}$ wavelet matrix such that when applied to a vector that is formed by stacking the columns of a 2D map, it results in the vector representation of the $2 \mathrm{D}$ wavelet transform of the map. We have $\Psi_{f, w} \triangleq \Phi_{\mathrm{pt}} \times \Gamma_{w}$.

\subsubsection{Impact of Nonideal Frequency Sampling}

So far, we discussed sampling in the frequency domain using the Fourier Slice Theorem. However, this theorem is for the case where we have continuous signals. For the case of a sampled signal (which is the case with our obstacle mapping), the theorem becomes an approximation. The quality of the approximation will then depend on the resolution of the sampled 2D signal and projections. We use the term nonideal frequency sampling to differentiate this realistic case from the case where the frequency samples are ideally available through the Fourier Slice Theorem. Let $g(\theta, t), G_{f}(\theta, f)$, $h(\theta, t)$, and $H_{t}(\theta, f)$ be the continuous signals, expressed in polar coordinates, as defined in the previous sections. Let $h_{s}(\theta, t)$ represent samples of $h(\theta, t)$, acquired through wireless measurements at step intervals of $\Delta: h_{s}(\theta, t)=$ $\sum_{n} h(\theta, t=n \Delta) \delta(t-n \Delta)$, where $\delta($.$) is the impulse func-$ tion. Let $\hat{H}_{t}(\theta, f)$ represent the Fourier transformation of this sampled signal. We have

$$
\hat{H}_{t}(\theta, f)=\sum_{n} h(\theta, t=n \Delta) e^{-j 2 \pi f n \Delta}=\frac{1}{\Delta} \sum_{n} H_{t}\left(\theta, f-\frac{n}{\Delta}\right) .
$$

Thus, to prevent aliasing, we need $\Delta \leq \frac{1}{2 \Omega_{f \theta}}$, where $\Omega_{f, \theta}$ is the bandwidth of the corresponding continuous function at that angle $(h(\theta, t))$. Similarly, let $g_{s}$ denote the sampled version of $g$, using a 2D impulse train. We have

$$
\begin{aligned}
\hat{G}_{f}\left(f_{i}, f_{q}\right) & =\sum_{n} \sum_{m} g(i=n \Delta, q=m \Delta) e^{-j 2 \pi n \Delta f_{i}-j 2 \pi m \Delta f_{q}} \\
& =\frac{1}{\Delta^{2}} \sum_{n} \sum_{m} G_{f}\left(f_{i}-\frac{n}{\Delta}, f_{q}-\frac{m}{\Delta}\right),
\end{aligned}
$$

where $\hat{G}_{f}\left(f_{i}, f_{q}\right)$ is the Fourier transformation of $g_{s}$, expressed in the Cartesian coordinates and $g(i=n \Delta, q=m \Delta)$ 
is the original map calculated at the inphase and quadrature components of $n \Delta$ and $m \Delta$, respectively. In order to prevent aliasing, we need $\Delta \leq \frac{1}{2 \Omega}$, where $\Omega$ represents the bandwidth of the continuous $2 \mathrm{D}$ obstacle map.

Wireless measurements will result in measuring $\hat{H}_{t}(\theta, f)$. Then, that is related to the 2D Fourier of the sampled 2D map through approximation, using the Fourier Slice Theorem: $\hat{H}_{t}(\theta, f) \approx \hat{G}_{f}(\theta, f)$ for samples of $f \in\left[\frac{-1}{2 \Delta}, \frac{1}{2 \Delta}\right)$. Since the map (and any projection) is space limited, it cannot be bandlimited and as such, there will always be aliasing. Then, the smaller $\Delta$ is, the better the quality of this approximation will be. For instance, (5) is written under the assumption that aliasing is negligible. In the next sections, we show the impact of nonideal frequency sampling on mapping quality.

\subsection{Coordinated Wireless Measurements and Space Sampling}

In the previous section, we discussed collecting samples of the frequency response of the obstacle map, using coordinated wireless measurements. Alternatively, the coordinated measurements can be used for direct space sampling and reconstruction. Let $y_{s, c}$ denote a vector that contains the acquired coordinated samples of $h(\theta, t)$, from (4). We have

$y_{s, c}=\Psi_{s, c} V_{g_{s}}$ coordinated space sampling and TV sparsity.

In each row of $\Psi_{s, c}$, the nonzero elements correspond to the obstacle map pixels that the corresponding ray visited, with each nonzero value indicating the distance traveled in the corresponding pixel. This matrix may not have good RIC properties, which can result in a poor performance if we use sparsity in the space domain (same is the case for the formulation in the wavelet domain). As such, a better way of solving for the obstacle map is by using the sparsity in $\mathrm{TV}$, as we shall show in the next section.

\subsection{Random Wireless Measurements and Space Sampling}

Consider Fig. 1 (right), where pairs of robots are making wireless measurements. In this case, we do not assume that the robots are attempting to have a specific pattern, i.e., the $\theta$ and $t$ can be chosen randomly. Thus, for a given $\theta$ and $t$ pair, a wireless transmission and reception is made, which results in measuring a line integral of (3). Let $y_{s, r}$ denote a vector that contains all the acquired random wireless measurements. Then, (8) can be modified to the following:

$$
y_{s, r}=\Psi_{s, r} V_{g_{s}} \text { random space sampling and TV sparsity, }
$$

where matrix $\Psi_{s, r}$ is similar to Matrix $\Psi_{s, c}$, except that it has no specific pattern. We can similarly solve for the obstacle map using TV sparsity.

The random space sampling approach is, in particular, useful for scenarios that the robots cannot make coordinated measurements, due to environmental constraints.

\subsection{Underlying Tradeoffs of Our Sampling and Reconstruction Techniques}

In this section, we show the performance of our framework, for compressive obstacle mapping, and shed light on the underlying tradeoffs of the aforementioned sampling and reconstruction techniques. As mentioned earlier, an obstacle map is typically considerably sparse in both space and

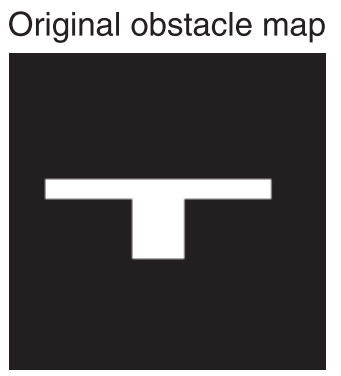

Wavelet transform

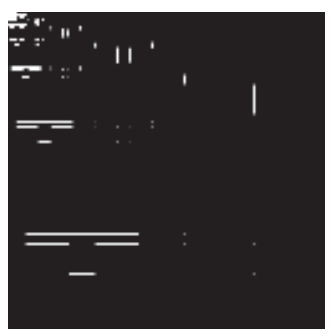

Fig. 3. A T-shaped obstacle map with the obstacle areas denoted in white, where 100 percent of the energy is in 7.52 percent of the space samples (left), and its transformed representation in the wavelet domain, where 100 percent of energy is in less than 1.82 percent of the coefficients (right).

wavelet domains. Furthermore, its spatial variations, measured by its Total Variation, are also considerably sparse. To see this, Fig. 3 (left) shows a T-shaped obstacle map. This is a horizontal cut of a real obstacle (see Fig. 9, first row), which we will use later for showing the performance of our framework with real data. As such, we use this T-shaped obstacle for the purpose of analysis and comparison throughout the paper. Fig. 3 (right) shows the 2D wavelet transform of the obstacle map, using Haar wavelets. As can be seen, the map is sparse in both domains, i.e., it can be represented by only a small percentage of the coefficients. More specifically, in the wavelet domain, this map can be represented with only 1.82 percent of the coefficients as compared to 7.52 percent in the space domain. While it is hard to mathematically prove the higher sparsity of the wavelet domain, for a general obstacle map, our analysis of several other obstacle maps consistently asserted this hypothesis. Alternatively, an obstacle map is also considerably sparse in its spatial variations. For instance, consider nonzero changes in both $\mathrm{x}$ and $\mathrm{y}$ directions. Clearly, the number of changes is less than the direct number of nonzero values in the space domain. It is hard, however, to establish any general comparison with the sparsity in the wavelet domain. Our analysis of several maps shows that utilizing the sparsity in spatial variations results in efficient obstacle mapping, as we shall explore in this section.

In this section, we start with the case of sampling in the frequency domain (ideal sampling), using coordinated wireless measurements and Fourier Slice Projection Theorem, as discussed in the previous section. Fig. 4 compares the performance of different reconstruction techniques (OMP, ROMP, and SPARSA) for the case of frequency sampling when using the sparsity in the space domain. The figure shows the Normalized Mean Square Error (NMSE) of the reconstruction of the T-shape of Fig. 3 (left), as a function of the percentage of the measurements collected in the frequency domain. NMSE is the Mean Square Error of the reconstruction divided by the power of the original signal of interest. Alternatively, the $x$-axis could be represented in terms of the number of angle measurements collected. ${ }^{2}$ For instance, the case of one angle indicates that two robots moved parallel to each other for only one given angle ( $\theta$ of Fig. 1) and made wireless measurements at different $t$ s along that angle. As a reference point, the case

2. Throughout the paper, we may represent the performance as a function of the sampling rate or the number of utilized angles, depending on the context. 


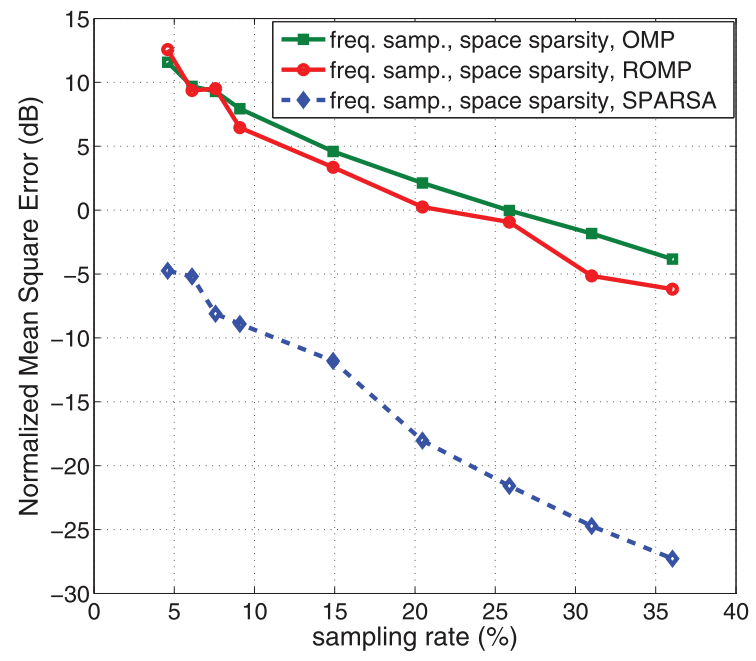

Fig. 4. Performance of different reconstruction techniques, based on Fourier sampling and space sparsity. As can be seen, SPARSA outperforms the MP approaches considerably. It also has a much lower computational complexity, as compared to OMP, and a comparable complexity to ROMP.

of 12 angles results in sampling only 9.09 percent of the Fourier transformation of this map. ${ }^{3}$ As such, without the compressive sensing framework, proper reconstruction would require prohibitive number of measurements. As can be seen from the figure, the MP approaches perform considerably worse than the BP approach using SPARSA. This is expected as MP approaches are simpler alternatives (but at the cost of a possible loss of performance) to the original $\ell_{1}$ relaxation method. In between MP approaches, OMP performs worse than ROMP as it is aimed at catching only one nonzero coefficient in every iteration (see Section 2.2). In terms of computational complexity, OMP also takes considerably longer than the other two approaches. For instance, for the case of 9.09 percent sampling rate, OMP takes 5.7 seconds to solve for the map on an Intel $3 \mathrm{GHz}$ Core 2 Duo E8400 processor with 2 GB of RAM, as compared to 0.35 seconds for ROMP or 1.8 seconds for SPARSA. As such, OMP is not a suitable compressive reconstruction technique for obstacle mapping. While the $\mathrm{BP}$ approaches are typically computationally more complex than ROMP, the recently proposed SPARSA is very efficient with a computational complexity comparable to ROMP. As such, it is a possible efficient technique for compressive reconstruction of an obstacle map.

Next, we consider frequency sampling and reconstruction using the sparsity in the wavelet domain (see (7)). We expect to gain a considerable performance improvement as the sparsity in the wavelet domain is noticeably higher than the space domain (see Fig. 3). The solid and dashed curves of Fig. 5 compare the performance of SPARSA reconstruction based on using the sparsity in the space and wavelet domains, respectively. As can be seen, the performance improves drastically when considering the sparsity in the wavelet domain. This, however, comes at the cost of a nonnegligible increase in computational complexity as the corresponding function handles have to deal with wavelet transformation. For instance, at 9.09 percent sampling rate,

3. Note that the frequency samples are uniformly spaced across each angle and the angles are uniformly spaced.

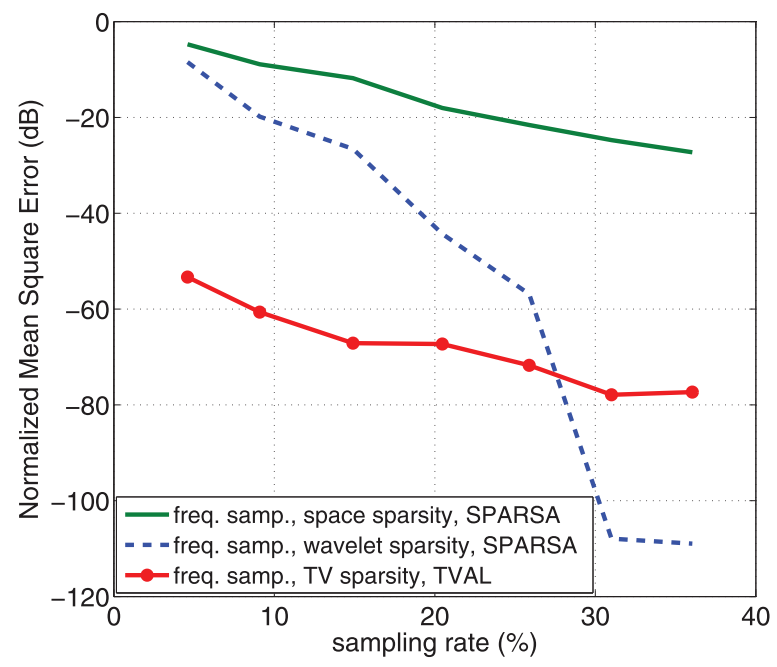

Fig. 5. Performance of different sparsity approaches, based on frequency sampling. The figure compares the performance of reconstruction based on utilizing the sparsity in space, wavelet and total variation (TV). As can be seen, using the sparsity in the spatial Total Variation provides the best performance for the most part. This is then followed by using the sparsity in the wavelet domain.

SPARSA wavelet takes 93.4 seconds while SPARSA space takes 1.8 seconds, on the same machine with the specifications mentioned above.

The last possibility for the case of frequency sampling is to use the sparsity in the spatial variations for reconstruction. The solid-circle curve of Fig. 5 shows the performance of this case, using a TVAL-based solver [27]. As can be seen, this approach results in a further performance improvement. Only at considerably high sampling rates, wavelet sparsity approach outperforms this case. Furthermore, with the newly proposed TVAL [27], the computational complexity of this approach is considerably less than both SPARSA wavelet and space approaches (0.289 seconds as compared to the aforementioned numbers). We consistently see the aforementioned observations with several other obstacle maps. As such, we find frequency sampling and reconstruction based on the sparsity of the spatial variations a viable candidate for compressive obstacle mapping. Using the sparsity in the wavelet domain is also another possibility, especially if the computational complexity is not a concern. Fig. 6 compares the reconstructed map for the aforementioned techniques, for the case where only 9.09 percent of the $2 \mathrm{D}$ Fourier function is sampled, using the Fourier Slice Theorem. As can be seen, the TV approach performs the best. This is followed by the wavelet-based approaches, in particular BP reconstruction with SPARSA. ROMP approach and wavelet sparsity can also produce a recognizable map. The ROMP-based space approach, on the other hand, results in a map that is composed of points as it attempts to directly capture the nonzero space values.

So far, we considered the performance of different reconstruction techniques and sparsity approaches for the case of Fourier sampling. Next, we compare the performance of different sampling techniques, i.e., Fourier, coordinated-space and random-space approaches. For coordinated space and Fourier sampling cases, equally-spaced angles are chosen with 0 degree included. The solid and dashed lines of Fig. 7 show the performance of coordinated and random space sampling approaches, respectively, for 


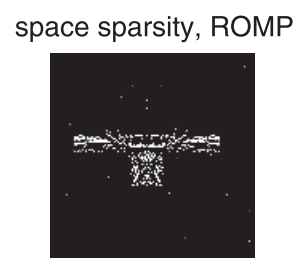

space sparsity, SPARSA

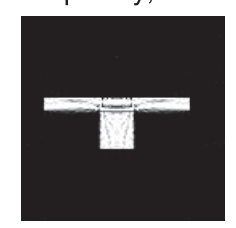

wavelet sparsity, ROMP

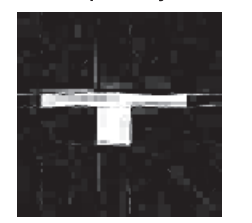

wavelet sparsity, SPARSA

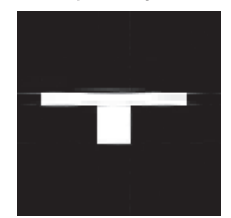

TV sparsity, TVAL

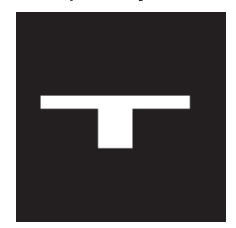

Fig. 6. The reconstructed obstacle map for the case of frequency sampling when only 9.09 percent of the Fourier function is sampled. The figure compares the performance of different sparsity/reconstruction techniques.

the obstacle map of Fig. 3 (left). For the coordinated space case, the $x$-axis can be thought of as being similar to the frequency sampling case, i.e., coordinated measurements along a number of angles are collected. The total number of coordinated transmissions/receptions along these angles then results in an equivalent percentage of the overall map size in pixels (the quoted sampling rate). Then, for the random space case, the same number of transmissions/ receptions is randomly gathered. This number can also be thought of as an equivalent number of frequency samples for comparison. As can be seen, the coordinated approach outperforms the random one considerably, for the range of demonstrated sampling rates, as expected. In terms of computational complexity, the coordinated space case has a noticeably lower complexity than the random one. ${ }^{4}$

Finally, the figure shows the performance with ideal frequency sampling. As can be seen, if the frequency samples can be selected perfectly, the performance is considerably better than the space approaches. In reality, however, this will not be the case as we discussed in Section 4.1.3. In the next section, we show the impact of nonideal frequency sampling when dealing with real data. As we shall see, the performance of the frequency sampling case becomes comparable to the coordinated space approach for real data.

\section{Compressive Cooperative Reconstruction of a Real Obstacle}

In the previous sections, we showed the performance of our framework in a simulation environment. In this section, we show its performance in constructing real obstacles, based

4. Note that the choice of the measurement angles can make a difference for the coordinated-space case. Further exploration of this is the subject of our future work.

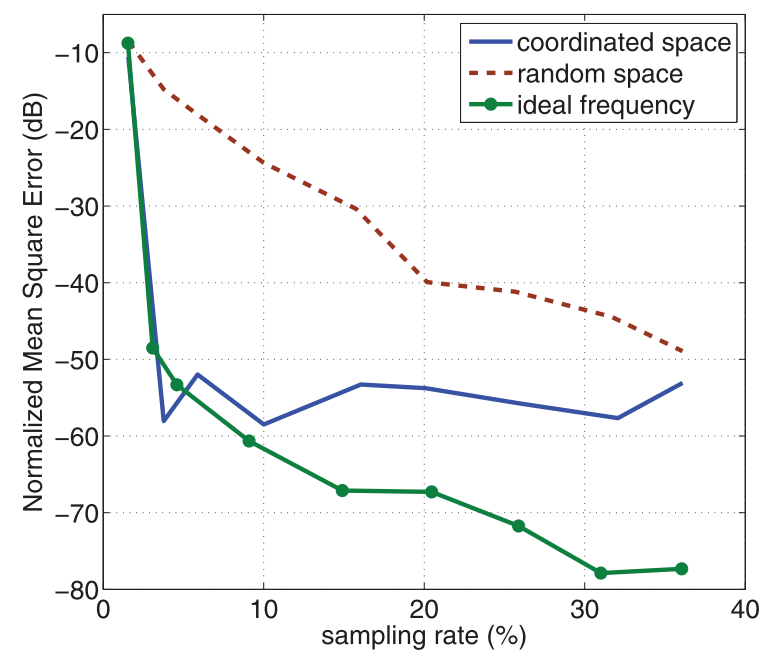

Fig. 7. A comparison of different proposed sampling techniques. All the reconstructions are with TVAL.

on a very small number of wireless channel measurements. Since the aforementioned modeling of a wireless channel cannot possibly embrace all the propagation phenomena, we do not expect perfect recovery with a very small number of measurements in a real environment. However, as long as the reconstruction is informative for the cooperative operation of a mobile network, it would be valuable. For instance, if it allows the vehicles to build a rough map of inside a room before entering it, it can be considerably useful. In this section, we show that it is indeed possible to map real obstacles and see through objects with a good quality, using our proposed framework.

In order to test our framework, we built an experimental setup for cooperative obstacle mapping. In this setup, a number of robots that are equipped with transceivers, make a small number of wireless channel measurements, as shown in this paper. Before we show the reconstruction performance, we briefly summarize our experimental platform and its key enabling features.

\subsection{A Summary of the Enabling Experimental Platform}

The advent of robotic networks facilitates the design of an automated cooperative mapping system and allows for collecting wireless measurements with flexibility, reconfigurability, and a high spatial resolution. As such, we have developed a robotic testbed for cooperative compressive obstacle mapping. There are two key enabling factors that contribute to the success of our experimental setup: 1) use of robotic units, which enables automated positioning for collecting wireless measurements and 2) use of adaptive directional narrow-beam antennas. The latter is crucial in limiting the impact of multipath fading, as we showed in [35]. As can be seen from (3), our mapping framework is based on following the shadowing component. More specifically, the shadowing component carries information on the obstacles, which we have utilized in our framework. As such, multipath fading appears as additional noise and can ruin the mapping quality. Therefore, in this paper we use adaptive directional antennas with narrow beamwidth, for cooperative obstacle mapping, in order to limit the impact of multipath fading in practice. 

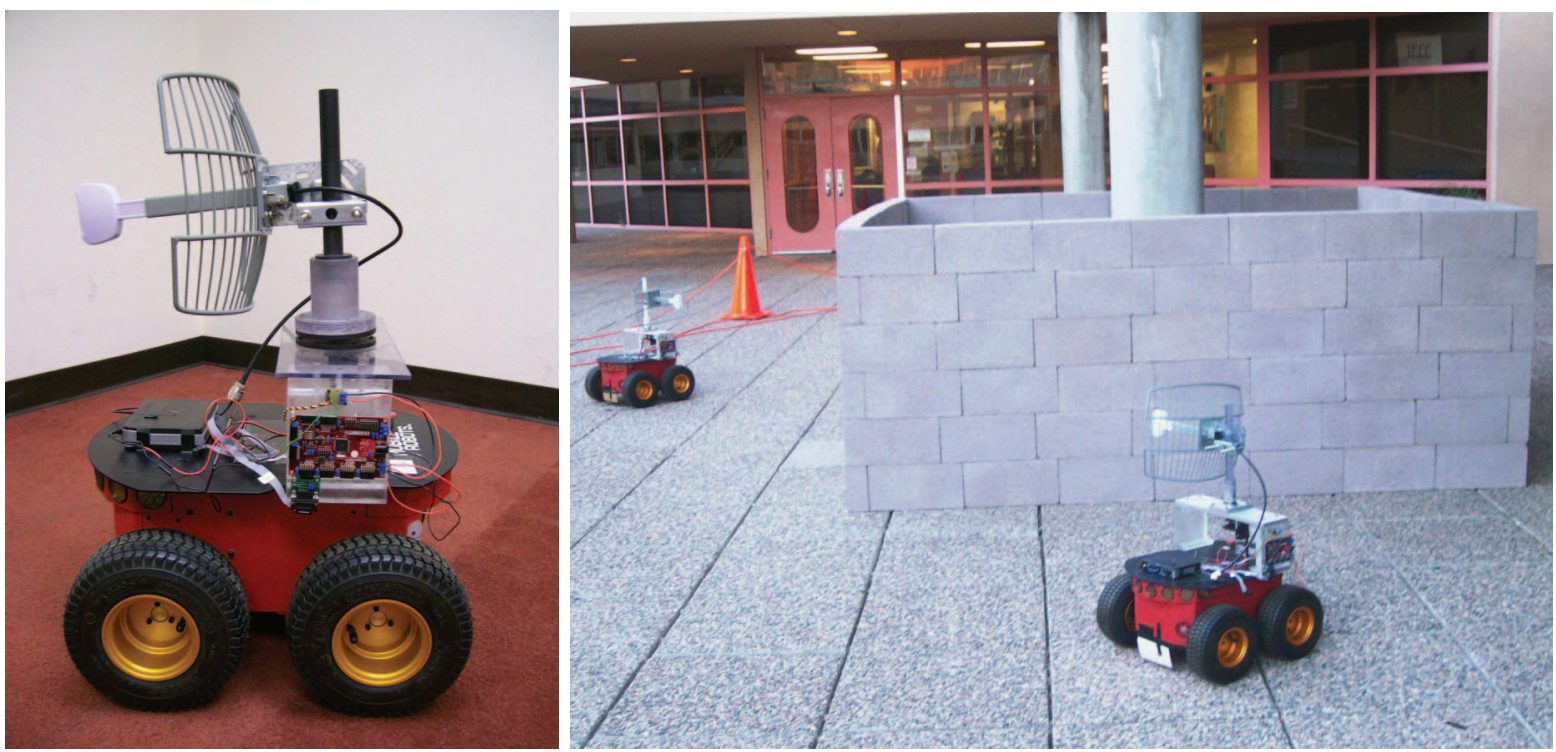

Fig. 8. A pioneer 3-AT robot equipped with our servo control mechanism/fixture and an adaptive narrow-beam directional antenna (left), two robots in action, making wireless measurements in order to map the obstacle (right). In the right figure, the outer structure is a square with a side of 2.86 meters while the inner column has a diameter of 0.5 meters.

Our setup consists of a number of Pioneer P3-AT mobile robots [36]. Pioneer 3-AT is a high performance robotic platform from MobileRobots, which is a popular and reliable team performer for indoor, outdoor, and roughterrain projects. Each robot is equipped with a wireless card and can record the received signal strength as it moves. We equipped each robot with a removable electromechanical fixture to hold a directional antenna. Fig. 8 (left) shows the resulting platform with the directional antenna mounted. We furthermore developed a servo motor control mechanism in order to intelligently rotate the directional antenna of the robot, such that it can point at any desired direction. ${ }^{5}$ As for the directional antennas, we use a GD24-15 $2.4 \mathrm{GHz}$ parabolic grid antenna from Laird Technologies [37]. This model has a $15 \mathrm{dBi}$ gain with 21 degree horizontal and 17 degree vertical beamwidth and is suitable for IEEE $802.11 \mathrm{~b} / \mathrm{g}$ applications. ${ }^{6}$

Each robot is furthermore equipped with the proper hardware and software for localization. More specifically, each robot uses its onboard gyroscope and wheel encoders for localization. The localization error is $2.5 \mathrm{~cm}$ for each traveled $1 \mathrm{~m}$, on the surface of the experiment site. The size of each route is less than 3 meters, for the experiments reported in this section. For each route, we manually correct any noticeable positioning error every $1 \mathrm{~m}$ (once or twice in the route). If we do not make any corrections for the whole route, we expect similar results since each current route is not that long and multipath fading is the main cause of performance degradation. A better understanding of the impact of localization errors on antenna alignment and the overall performance, however, is definitely important and is a subject of our future work. This is, in particular, important for operation over larger areas. Also better localization methods from the robotics literature can be used.

5. Note that rotating the angle is not necessary for the frequency sampling or coordinated space sampling case.

6. Note that the beamwidth of the antenna is fixed, but with the aforementioned electromechanical fixture, the antenna can rotate to point at any direction.
Two robots can then use the position information of each other (which is prone to localization errors) in order to align their antennas. ${ }^{7}$ It should be noted that for the cases of frequency sampling and coordinated space sampling, the robots start at each motion angle by pointing toward each other (each antenna perpendicular to the route direction that the robot is supposed to traverse and toward the structure that it is supposed to map) and do not need to change their antenna angles for the entire experiment. Next, we show the performance of our mapping framework, using this testbed.

\subsection{Cooperative Mapping of Real Obstacles Using Our Proposed Framework}

We tested our framework with our experimental setup, where two of our robots made a small number of wireless measurements cooperatively, in order to build a 2D map of a number of obstacles. In this section, we show the performance for the three obstacles of Fig. 9 (left column). Horizontal cuts (2D map) of these obstacles are shown in the right column of Fig. 9. Our robots then aim at reconstructing these structures, based on only wireless channel measurements. In this paper, we consider reconstruction in a horizontal plane, i.e., the goal is for the robots to map the horizontal cuts of Fig. 9 (right column). Fig. 10 shows the mapping quality as a function of the number of utilized angles. ${ }^{8}$ In this figure, all the reconstructions are based on coordinated space sampling, i.e., two robots move along a number of angles in parallel, as shown in Fig. 1 (left). As we observed from the previous section, coordinated space or frequency sampling and TV sparsity provided the best overall performance and computational complexity for most cases. As such, we used coordinated space sampling, sparsity in the spatial variations (TV) and TVAL for reconstruction in this figure. Later in this section,

7. As part of our future work, we plan to also use the feedback from the received communication signal strength for further antenna alignment

8. In order to avoid printing artifacts, it is best to also view all the reconstructed real obstacles in the pdf file. 


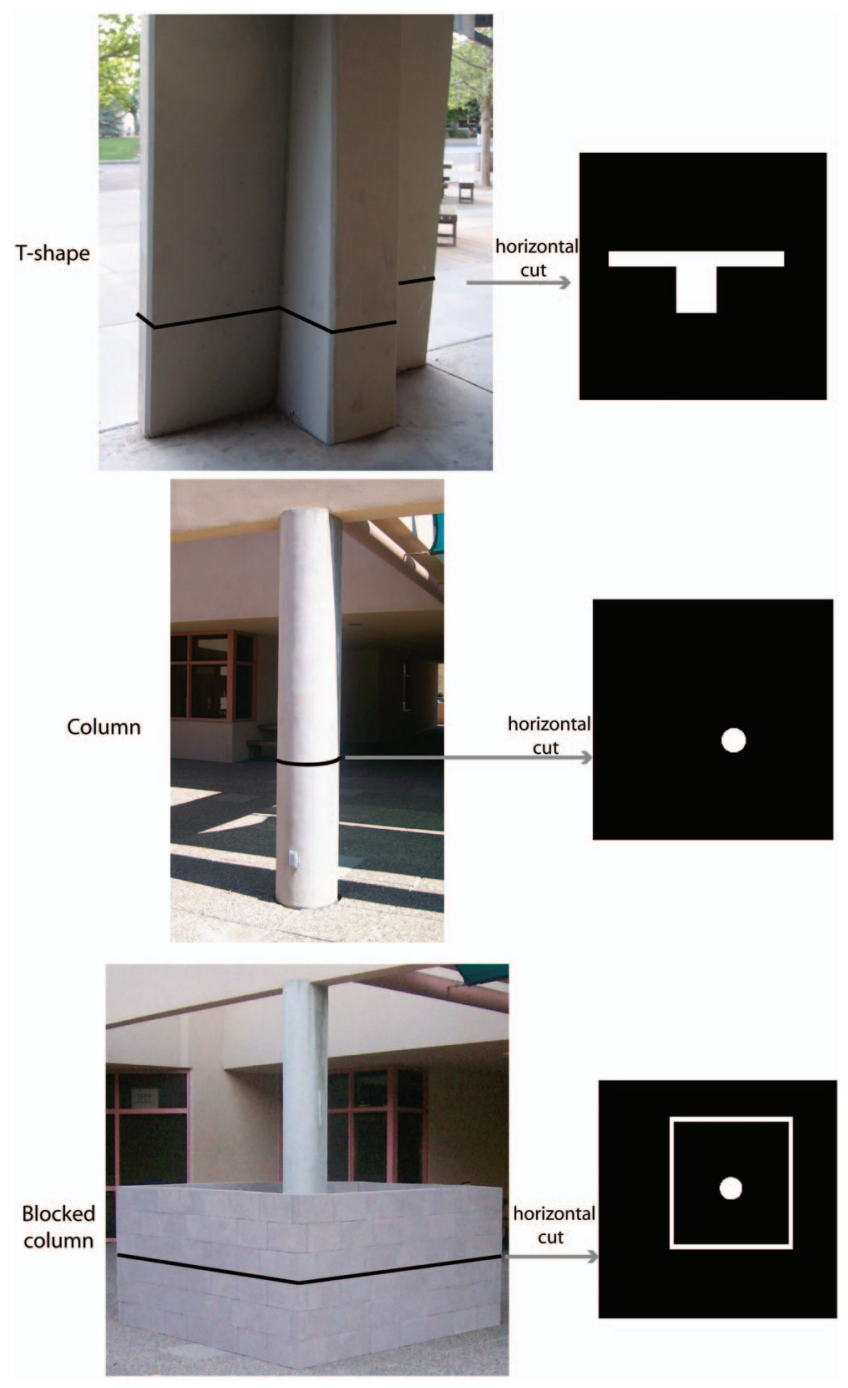

Fig. 9. The figures show a T-shaped column, a circular column and a blocked column. Horizontal cuts of these structures are also shown. Our robots aim to reconstruct the horizontal cut, using our framework.

we discuss the underlying tradeoffs with other sampling and reconstruction techniques for real data.

The first row of Fig. 10 shows the reconstruction of the T-shaped structure, for different total number of utilized angles. For each total number of angles, uniformly-spaced angles are chosen (with zero degree included). For instance, the first case of four total angles means that the robots made coordinated wireless measurements, by moving along four pairs of parallel lines. These lines have the angles of $0,90,45$, and 135 degrees with respect to the $x$ axis in Fig. 1 (left). For comparison with the previous results, where we discussed the performance as a function of the percentage of the sampled points, the case of four angles is equivalent to the sampling rate of 3.03 percent, which is considerably low. As can be seen, by making more coordinated measurements at six angles, the performance improves considerably. The improvement is slightly less from six to 12 angles, as expected. Adding more measurements at a low sampling rate can typically result in a more drastic improvement. Overall, the reconstructions are noisy as expected, due to several propagation phenomena that our modeling did not include. However, the T-shaped structure, with all its details, can be easily seen. In terms of NMSE, the case of 12 angles, for instance, results in an NMSE of $-3.78 \mathrm{~dB}$.

The second row shows the mapping performance for the column structure of Fig. 9. In this case, if only two angles are used ( 0 and 90 degrees), this object will not be fully observable, even if the wireless measurements were perfect with no multipath fading/noise. By not fully observable, we mean that some details of the object will not be detectable even in the perfect sampling scenario. For instance, in the case of two angles, this obstacle will be reconstructed as a square in the best case. For the case of real measurements, as can be seen, our framework tries to reconstruct a square for the case of two angles. As we increase the number of angles to $4(0,90,45$, and 135 degrees), then the reconstruction attempts to map the curvatures as well. Similar to the previous case, four angles only results in 3.03 percent sampling rate, which is considerably low. Still, the structure can be correctly identified in the right location, even with such small number of measurements. Depending on the application, the robots may not have time to make several wireless measurements. As such, the capability to identify and map structures, with such small sampling rates, can be useful.

Finally, the last row shows the performance in mapping the blocked-column structure of Fig. 9, for four and eight equally-spaced angles including 0 degree. As can be seen, the reconstruction is noisy with four angles, while increasing the angles to eight can considerably improve the mapping quality. It is important to note how the robots can see through the walls and correctly map the column inside for this case, which is an important feature of the proposed framework. Also, note that this structure is not homogeneous, i.e., the inside column and the walls are made out of two different materials.

Fig. 11 shows the case where a threshold is applied to three of the reconstructed maps of Fig. 10, such that any value that is $10 \mathrm{~dB}$ below the maximum is zeroed. This was done because we noticed that there could be scenarios where reconstructed pixels with very small values get magnified by some printers or monitors with certain gamma settings. A simple thresholding can avoid such cases. In general, it is best to also check the pdf file in order to avoid other printing artifacts.

So far, we showed the performance of our coordinated space sampling approach in mapping real obstacles. Next, we consider the performance of the frequency sampling approach. As we saw from Fig. 7, if the frequency samples could be chosen perfectly, then the frequency approach outperforms any space approach considerably. However, in reality, there will be a loss of performance due to the fact that the Fourier Slice Theorem, while fully holding for continuous functions or proper sampling of bandlimited signals, becomes an approximation when sampling spacelimited signals. The quality of this approximation depends on the sampling resolution and the frequency response of the original map. Fig. 12 shows the mapping quality of the frequency approach for two of the structures of Fig. 9. As can be seen, the reconstruction quality is almost the same as that of Fig. 10 with coordinated space measurements (we note that it is not exactly the same). In general, we observed that frequency sampling results in a reconstruction very similar to the coordinated space approach, when dealing with real data. However, it has a higher computational 

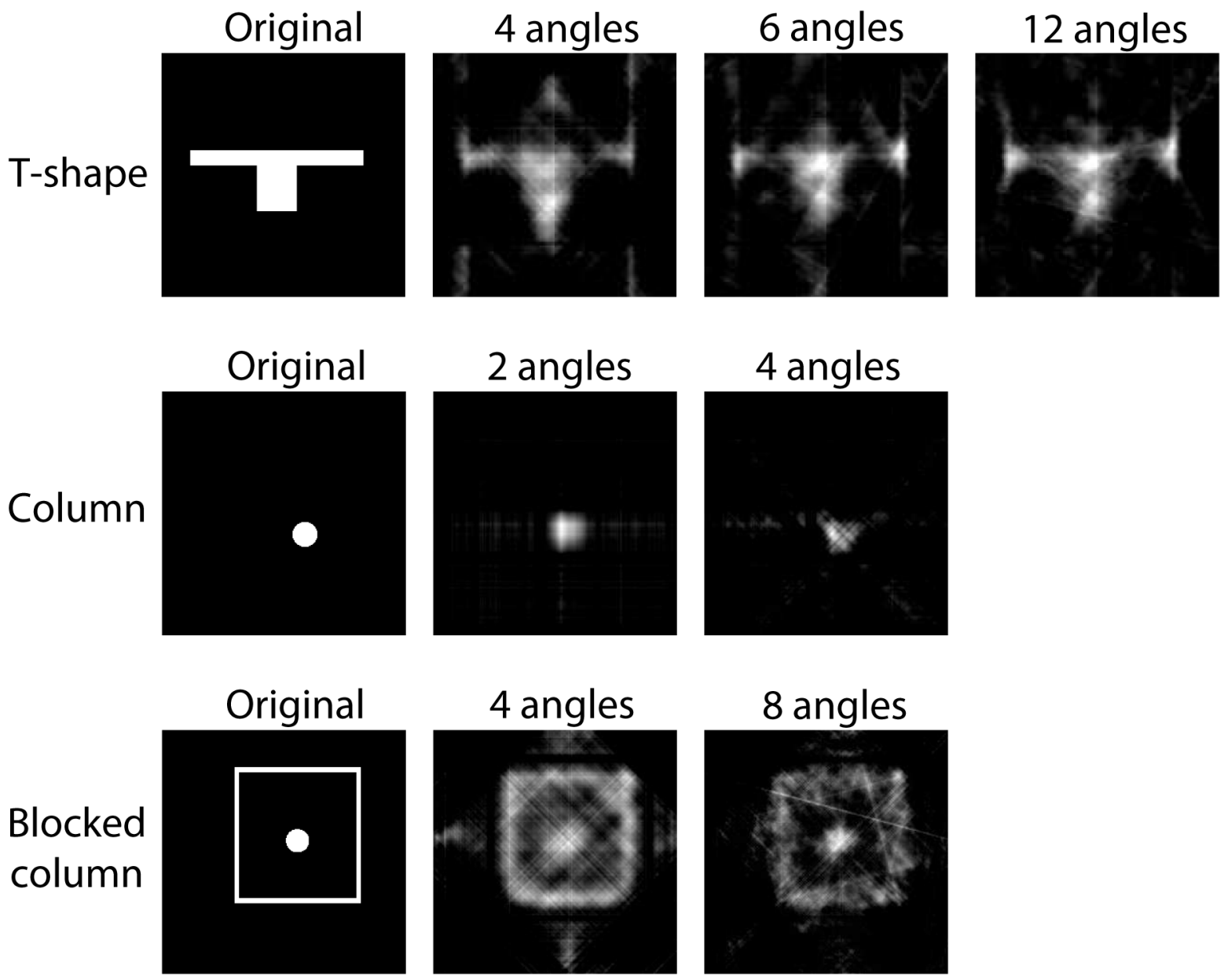

Fig. 10. Performance of our proposed framework in mapping three real structures. The reconstructions are based on coordinated space sampling and TV sparsity. The three structures and their horizontal cuts are shown in Fig. 9. As can be seen, the original structures and their details can clearly be seen in our reconstruction although very few wireless measurements were taken.

complexity. For instance, the coordinated space sampling and TVAL takes 20.7 seconds to solve (in post processing after the measurements are collected), for the T-shaped structure of Fig. 10 at 12 angles, while reconstruction based on frequency sampling in Fig. 12 takes 722.8 seconds, for the same measurements.

Finally, we briefly compare the performance of our coordinated and random space sampling approaches in mapping real obstacles. As we showed through simulations in Section 4.4, the coordinated approach outperforms the random one considerably. However, if the coordinated approach can only make measurements at extremely low number of angles, such as one, the random approach can possibly outperform the coordinated one. This makes sense as the coordinated approach can only sample the structure from limited views, for the case of one angle. The random approach, on the other hand, samples the map from possibly different views even at a considerably small sampling rate.

To see this in mapping a real obstacle, Fig. 13 shows the performance of the two approaches, in mapping the T-shaped structure of Fig. 9, for the case of one angle of 90 degree for the coordinated sampling (0.77 percent sampling rate), as well as for a random case with an equivalent percentage measurement. For the random case, the 0.77 percent gathered measurements are randomly chosen from the pool of available coordinated measurements for the case of six angles for this structure. As can be seen, for the case of random sampling, the structure is still visible, albeit noisy, whereas with coordinated measurements, the structure is not simply observable at this low rate. However, once we measure the structure from more angles, the coordinated approach can outperform the random one, as was discussed for the simulated data. Then, we have the following open question: Given a given number of possible available channel measurements, what are the optimum choice and number of angles over which the measurements should be distributed? This is a subject of our future papers, due to space limitations. As mentioned earlier, the complexity of the random case is typically higher, both in terms of antenna alignment and processing of the data. Thus, in practice, the coordinated case should be used, if possible, and the random case should be reserved for cases where the robots cannot physically make coordinated measurements, due to environmental constraints. In summary, we find coordinated-space sampling and frequency sampling approaches two promising techniques for obstacle mapping in a real environment (from a performance standpoint). Furthermore, sparsity in TV or wavelet can be utilized for reconstruction with minimal sensing. The random sampling case can then be utilized in scenarios where there are environmental navigation constraints. 


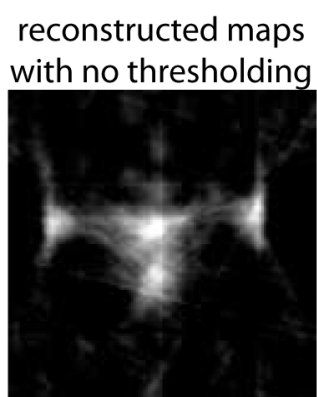

12 angles

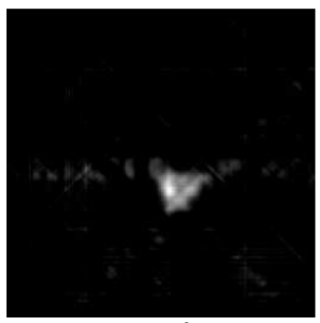

4 angles

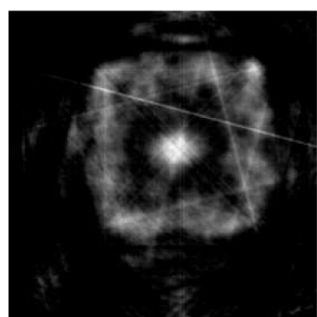

8 angles reconstructed maps

after thresholding

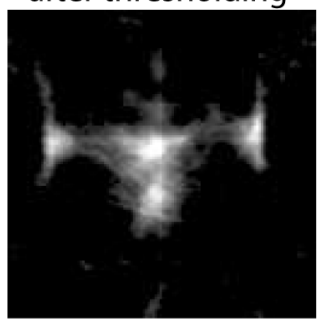

12 angles

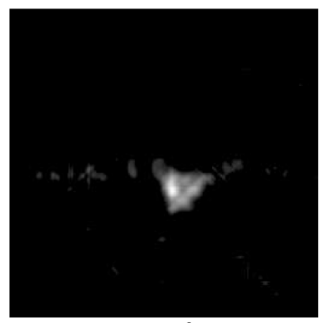

4 angles

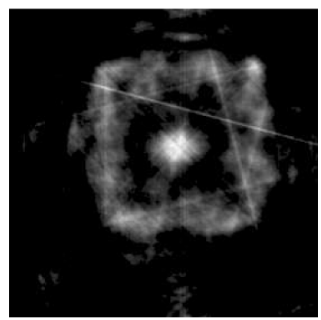

8 angles
Fig. 11. Mapping quality after a threshold of $10 \mathrm{~dB}$ is applied to three of the reconstructed maps of Fig. 10. The threshold is applied such that any value that is $10 \mathrm{~dB}$ below the maximum is zeroed. There could be scenarios where original reconstructed pixels with very small values get magnified by some printers or monitors with certain gamma settings. A simple thresholding can avoid such cases.

\section{Conclusions AND Further Extensions}

In this paper, we considered a mobile cooperative network that is tasked with building an obstacle map, including mapping of occluded obstacles. We developed a theoretical and experimental framework for the mapping of obstacles, based on a small number of wireless channel measurements. We considered three approaches based on coordinated space, random space, and frequency sampling, and showed how the robots can exploit the sparse representation of the map in space, wavelet or spatial variations, in order to build it with minimal sensing. We then discussed the underlying tradeoffs of all the possible sampling, sparsity, and reconstruction techniques. Our simulation and experimental results indicated the feasibility and good performance of the proposed framework. More specifically, we showed how to design an experimental robotic platform, in order to successfully implement the proposed approach and efficiently map a number of simple real obstacles, including an occluded one. Our results indicated that the coordinated space sampling or frequency sampling approaches, along with utilizing the sparsity in total variations or wavelet, are promising techniques (from a performance standpoint) for obstacle mapping in real environments. The

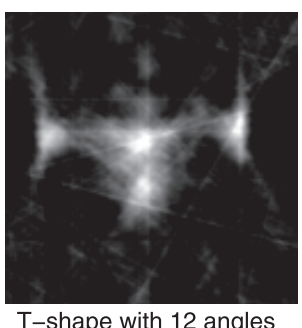

T-shape with 12 angles

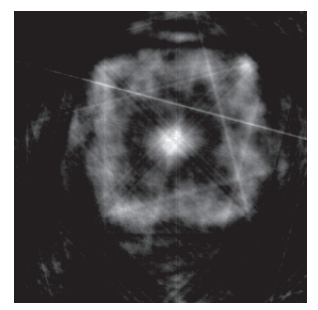

Blocked column with 8 angles
Fig. 12. Performance of the frequency sampling approach, based on TV sparsity. As can be seen, the reconstruction quality is very similar to that of Fig. 10, where coordinated measurements and space sampling were used.
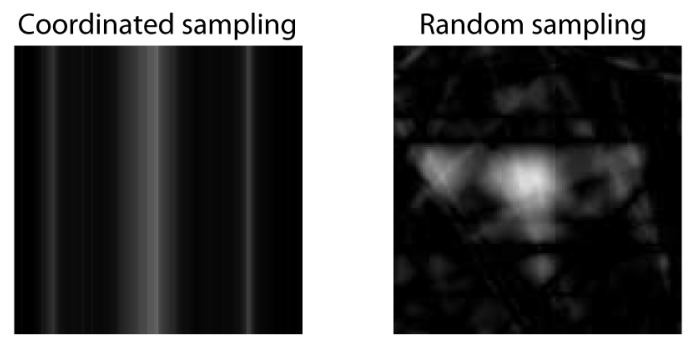

Fig. 13. Comparison of coordinated and random space sampling approaches in mapping a real obstacle at extremely low sampling rates. Both approaches attempt to build the T-shaped structure of Fig. 9, with only 0.77 percent measurements (only one angle for the coordinated case). As can be seen, at this extremely low rate, the random approach still results in a recognizable reconstruction, albeit noisy, while the structure becomes unrecognizable with the coordinated case. As the sampling rate increases (or the samples are divided among more angles at the same sampling rate), the coordinated case outperforms the random one, as we discussed in this paper.

random sampling case (possibly combined with the aforementioned techniques) can then be utilized in scenarios where there are environmental navigation constraints.

There are several possible extensions of this work. Here, we briefly discuss three important ones. In this paper, we considered reconstruction of simple structures in a 2D plane. We are currently working on extending the framework to 3D reconstructions. We expect to gain more from sparsity, when moving to 3D. We also discussed observability of a structure and the related minimum number of measurements required for its reconstruction. We are currently working on a mathematical characterization of this. This characterization will then allow us to systematically extend our framework to the mapping of more complicated obstacle structures and cluttered environments. Finally, we do not view this framework as a replacement for the current sensing and mapping techniques. We rather view it as a complement to the existing approaches, by providing a seethrough capability. Proper integration of this approach with the existing techniques is, therefore, an interesting extension of this work.

\section{ACKNOWLEDGMENTS}

The author would like to acknowledge the help of Alejandro Gonzalez-Ruiz with running the experiments. Furthermore, she would like to thank Alireza Ghaffarkhah for his help with developing the experimental setup, Soheil Darabi for useful discussions on the solvers, and Professor Pradeep Sen for useful technical discussions. This work was 
supported by US National Science Foundation CAREER award \# 0846483. A very small part of this work was presented at Milcom 2010 [1]. Compared to that conference paper, this paper develops a considerably more comprehensive framework by 1) proposing and comparing a number of possible sampling and sparsity approaches for obstacle mapping, based on wireless measurements, 2) showing how to develop the necessary experimental platform, and 3) demonstrating the performance for a number of real obstacles (including an occluded one).

\section{REFERENCES}

[1] Y. Mostofi and A. Gonzales-Ruiz, "Compressive Cooperative Obstacle Mapping," Proc. Military Comm. (Milcom), Oct. 2010.

[2] R. Murray, Control in an Information Rich World: Report of the Panel on Future Directions in Control, Dynamics, and Systems. SIAM, 2003.

[3] C. Chong and S. Kumar, "Sensor Networks: Evolution, Opportunities and Challenges," Proc. IEEE, vol. 91, no. 8, pp. 1247-1256, Aug. 2003.

[4] B. Sinopoli, C. Sharp, L. Schenato, S. Schaffert, and S. Sastry, "Distributed Control Applications within Sensor Networks," Proc. IEEE, vol. 91, no. 8, pp. 1237-1246, Aug. 2003.

[5] H. Durrant-Whyte and T. Bailey, "Simultaneous Localization and Mapping: Part I," IEEE Robotics and Automation Magazine, vol. 13, no. 2, pp. 99-110, June 2006.

[6] T. Bailey and H. Durrant-Whyte, "Simultaneous Localization and Mapping: Part II," IEEE Robotics and Automation Magazine, vol. 13, no. 3, pp. 108-117, Sept. 2006.

[7] H. Choset and K. Nagatani, "Topological Simultaneous Localization and Mapping (SLAM): Toward Exact Localization without Explicit Localization," IEEE Trans. Robotics and Automation, vol. 17, no. 2, pp. 125-137, Apr. 2001

[8] S. Thrun, D. Koller, A. Ng, Z. Ghahramani, and H. DurrantWhyte, "Simultaneous Localization and Mapping with Sparse Extended Information Filters," Int'l J. Robotics Research, vol. 23, nos. 7/8, pp. 693-716, Aug. 2004.

[9] R. Gartshore, A. Aguado, and C. Galambos, "Incremental Map Building Using an Occupancy Grid for an Autonomous Monocular Robot," Proc. Seventh Int'l Conf. Control, Automation, Robotics and Vision, vol. 2, pp. 613-618, Dec. 2002.

[10] H. Gonzalez-Banos and J.C. Latombe, "Navigation Strategies for Exploring Indoor Environments," The Int'l J. Robotics Research, vol. 21, nos. 10/11, pp. 829-848, 2002.

[11] R. Pito, "A Solution to the Next Best View Problem for Automated Surface Acquisition," IEEE Trans. Pattern Analysis and Machine Intelligence, vol. 21, no. 10, pp. 1016-1030, Oct. 1999.

[12] J.J. Kuffner and S.M. LaValle, "RRT-Connect: An Efficient Approach to Single-Query Path Planning," Proc. IEEE Int'l Conf. Robotics and Automation, Apr. 2000.

[13] A. Ganguli, J. Cortes, and F. Bullo, "Maximizing Visibility in Nonconvex Polygons: Nonsmooth Analysis and Gradient Algorithm Design," Proc. Am. Control Conf., June 2005.

[14] R. Grabowski, P. Khosla, and H. Choset, "Autonomous Exploration Via Regions of Interest," Proc. IEEE/RSJ Int'l Conf. Intelligent Robots and Systems (IROS '03), Oct. 2003.

[15] M.C. Wicks, "RF Tomography with Application to Ground Penetrating Radar," Proc. Asilomar Conf. Signals, Systems and Computers, pp. 2017-2022, Nov. 2007.

[16] E. Candès, J. Romberg, and T. Tao, "Robust Uncertainty Principles: Exact Signal Reconstruction from Highly Incomplete Frequency Information," IEEE Trans. Information Theory, vol. 52, no. 2, pp. 489-509, Feb. 2006

[17] D.L. Donoho, "Compressed Sensing," IEEE Trans. Information Theory, vol. 52, no. 4, pp. 1289-1306, Apr. 2006.

[18] J. Wilson and N. Patwari, "Radio Tomographic Imaging with Wireless Networks," IEEE Trans. Mobile Computing, vol. 9, no. 5, pp. 621-632, May 2010.

[19] M. Kanso and M. Rabbat, "Compressed RF Tomography for Wireless Sensor Networks: Centralized and Decentralized Approaches," Proc. IEEE Int'l Conf. Distributed Computing in Sensor Systems, June 2009.

[20] Y. Mostofi and P. Sen, "Compressive Cooperative Mapping in Mobile Networks," Proc. 28th Am. Control Conf. (ACC), pp. 33973404, June 2009.
[21] Y. Mostofi, "Compressive Cooperative Sensing and Mapping in Mobile Networks," IEEE Trans. Mobile Computing, vol. 10, no. 12, pp. 1770-1785, Dec. 2011.

[22] D. Needell and R. Vershynin, "Uniform Uncertainty Principle and Signal Recovery via Regularized Orthogonal Matching Pursuit," rapid post, 2007.

[23] S.J. Wright, R.D. Nowak, and M.A.T. Figueiredo, "Sparse Reconstruction by Separable Approximation," Proc. IEEE Int'l Conf. Acoustics, Speech and Signal Processing, pp. 3373-3376, Apr. 2008.

[24] J. Tropp and A. Gilbert, "Signal Recovery from Random Measurements via Orthogonal Matching Pursuit," IEEE Trans. Information Theory, vol. 53, no. 12, pp. 4655-4666, Dec. 2007.

[25] Y. Mostofi and P. Sen, "Compressed Mapping of Communication Signal Strength," Proc. IEEE Military Comm. Conf. (MILCOM), 2008.

[26] " $\ell_{1}$ Magic Toolbox," http://www.acm.caltech.edu/l1magic, 2012.

[27] C. Li, “An Efficient Algorithm for Total Variation Regularization with Applications to the Single Pixel Camera and Compressive Sensing," PhD thesis, RICE Univ., 2009.

[28] W.C. Jakes, Microwave Mobile Communication. Wiley-IEEE, 1994.

[29] Y. Mostofi, A. Gonzalez-Ruiz, A. Ghaffarkhah, and D. Li, "Characterization and Modeling of Wireless Channels for Networked Robotic and Control Systems - A Comprehensive Overview," Proc. IEEE/RSJ Int'l Conf. Intelligent Robots and Systems, 2009.

[30] A. Goldsmith, Wireless Communication. Cambridge Univ., 2005.

[31] A.C. Kak and M. Slaney, Principles of Computerized Tomographic Imaging. IEEE, 1988.

[32] R. Ketcham, Computed Tomography for Paleontology and Geology. Cambridge Univ., 2004.

[33] R. Ng, "Fourier Slice Photography," Proc. ACM SIGGRAPH, pp. 735-744, 2005.

[34] M. Rudelson and R. Vershynin, "Sparse Reconstruction by Convex Relaxation: Fourier and Gaussian Measurements," Proc. 40th Ann. Conf. Information Sciences and Systems, 2006.

[35] A. Gonzalez-Ruiz, A. Ghaffarkhah, and Y. Mostofi, "A Comprehensive Overview and Characterization of Wireless Channels for Networked Robotic and Control Systems," J. Robotics, vol. 2011, article 340372, 2011

[36] MobileRobots, Inc., http://www.mobilerobots.com, 2009.

[37] Laird Technologies, http://www.lairdtech.com/Products / Antennas-and-Reception-Solutions, 2010.

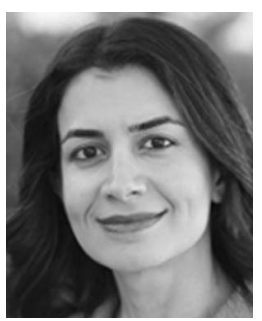

Yasamin Mostofi received the BS degree in electrical engineering from the Sharif University of Technology, Tehran, Iran, in 1997, and the $\mathrm{MS}$ and $\mathrm{PhD}$ degrees in the area of wireless communication systems from Stanford University, California, in 1999 and 2004, respectively. She is currently an associate professor in the Department of Electrical and Computer Engineering at the University of California Santa Barbara. Prior to that, she was a faculty member in the Department of Electrical and Computer Engineering at the University of New Mexico from 2006 to 2012. She was a postdoctoral scholar in control and dynamical systems at the California Institute of Technology from 2004 to 2006. Dr. Mostofi is the recipient of the Presidential Early Career Award for Scientists and Engineers (PECASE), the National Science Foundation (NSF) CAREER award, and the IEEE 2012 Outstanding Engineer Award of Region 6. She also received the Bellcore fellow-advisor award from the Stanford Center for Telecommunications in 1999 and the 2008-2009 Electrical and Computer Engineering Distinguished Researcher Award from the University of New Mexico. Her research is on mobile sensor networks. Current research thrusts include communication-aware navigation and decision making in robotic networks, compressive sensing and control, obstacle mapping, robotic routers, and cooperative information processing. She has served on the Control Systems Society conference editorial board since 2008. She is a member of the IEEE. 